\title{
Sistema computacional para dosimetria de nêutrons e fótons baseado em métodos estocásticos aplicado a radioterapia e radiologia*
}

Stochastic method-based computational system for neutron/photon dosimetry applied to radiotherapy and radiology

\author{
Bruno Machado Trindade ${ }^{1}$, Tarcisio Passos Ribeiro de Campos $^{2}$
}

Resumo Objetivo: Este artigo mostra um procedimento de conversão de imagens de tomografia computadorizada ou de ressonância magnética em modelo de voxels tridimensional para fim de dosimetria. Este modelo é uma representação personalizada do paciente que pode ser usado na simulação, via código MCNP (Monte Carlo N-Particle), de transporte de partículas nucleares, reproduzindo o processo estocástico de interação de partículas nucleares com os tecidos humanos. Materiais e Métodos: O sistema computacional desenvolvido, denominado SISCODES, é uma ferramenta para planejamento computacional tridimensional de tratamentos radioterápicos ou procedimentos radiológicos. Partindo de imagens tomográficas do paciente, o plano de tratamento é modelado e simulado. São então mostradas as doses absorvidas, por meio de curvas de isodoses superpostas ao modelo. O SISCODES acopla o modelo tridimensional ao código MCNP5, que simula o protocolo de exposição à radiação ionizante. Resultados: O SISCODES vem sendo utilizado no grupo de pesquisa NRI/CNPq na criação de modelos de voxels antropomórficos e antropométricos que são acoplados ao código MCNP para modelar braquiterapias e teleterapias aplicadas a tumores em pulmões, pelve, coluna, cabeça, pescoço, e outros. Os módulos atualmente desenvolvidos no SISCODES são apresentados junto com casos exemplos de planejamento radioterápico. Conclusão: O SISCODES provê de maneira rápida a criação de modelos de voxels personalizados de qualquer paciente que podem ser usados em simulações por códigos estocásticos tipo MCNP. A combinação da simulação via MCNP com um modelo personalizado do paciente traz grandes melhorias na dosimetria de tratamentos radioterápicos.

Unitermos: SISCODES; Radioterapia; Dosimetria; Dose absorvida; Teleterapia; Braquiterapia.

\begin{abstract}
Objective: The present paper describes a procedure for conversion of computed tomography or magnetic resonance images into a three-dimensional voxel model for dosimetry purposes. Such model is a personalized representation of the patient that can be utilized in nuclear particle transport simulations by means of the MCNP (Monte Carlo N-Particle) code, reproducing the stochastic process of nuclear particles interaction with human tissues. Materials and Methods: The developed computational system - SISCODES - is a tool designed for 3D planning of radiotherapy or radiological procedures. Based on tomographic images of the patient, the treatment plan is modeled and simulated. Then, the absorbed doses are shown by means of isodose curves superimposed on the model. The SISCODES couples the threedimensional model with the MCNP5 code, simulating the protocol of exposure to ionizing radiation. Results: The SISCODES has been utilized by the NRI/CNPq in the creation of anthropomorphic and anthropometric voxel models which are coupled with the MCNP code for modeling brachytherapy and teletherapy applied to lung, pelvis, spine, head and neck tumors, among others. The current SISCODES modules are presented together with examples of cases of radiotherapy planning. Conclusion: The SISCODES provides a fast method to create personalized voxel models of any patient which can be used in stochastic simulations. The combination of the MCNP simulation with a personalized model of the patient increases the dosimetry accuracy in radiotherapy.
\end{abstract}

Keywords: SISCODES; Radiotherapy; Dosimetry; Absorbed dose; Teletherapy; Brachytherapy.

Trindade BM, Campos TPR. Sistema computacional para dosimetria de nêutrons e fótons baseado em métodos estocásticos aplicado a radioterapia e radiologia. Radiol Bras. 2011 Mar/Abr;44(2):109-116.

* Trabalho realizado na Escola de Engenharia da Universidade Federal de Minas Gerais (UFMG), Belo Horizonte, MG, Brasil.

1. Mestre, Doutorando em Ciências e Técnicas Nucleares Programa de Pós-Graduação em Ciências e Técnicas Nucleares da Universidade Federal de Minas Gerais (UFMG), Professor do Centro Universitário Una, Belo Horizonte, MG, Brasil.

2. Doutor em Engenharia Nuclear, Professor Associado III do Curso de Pós-Graduação em Ciências e Técnicas Nucleares da
Universidade Federal de Minas Gerais (UFMG), Belo Horizonte, MG, Brasil.

Endereço para correspondência: Dr. Tarcisio Passos Ribeiro de Campos. Avenida Antonio Carlos, 6627, prédio PCA1, sala 2285, Campus Pampulha. Belo Horizonte, MG, Brasil, 31270-901. E-mail: campos@nuclear.ufmg.br

Recebido para publicação em 29/9/2010. Aceito, após revisão, em 17/2/2011.

\section{INTRODUÇÃO}

Radioterapia representa uma importante técnica de tratamento oncológico ${ }^{(\mathbf{1})}$. Nesta modalidade de tratamento, radiação ionizante é diretamente aplicada ao tumor de 
modo a, ao menos, retardar seu crescimento. Praticamente $50 \%$ dos pacientes com câncer serão submetidos à radioterapia, que pode ser usada como único tratamento ou ser associada a outras modalidades.

O método de Monte Carlo é aplicado para reproduzir um processo estatístico similar à interação de partículas nucleares com os tecidos humanos. Esta técnica é particularmente interessante na resolução de problemas complexos que não podem ser modelados por métodos computacionais determinísticos. No transporte de partículas nucleares, a técnica de Monte Carlo acompanha cada uma das muitas partículas primárias ou secundárias, geradas por uma fonte radioativa, durante sua vida até sua morte em algum evento terminal, tal como: absorção, espalhamento, fuga do sistema, entre outros ${ }^{(2)}$. A simulação tridimensional do transporte de partículas nucleares é uma importante ferramenta para a melhoria da qualidade dos procedimentos radioterápicos em oncologia ${ }^{(3)}$. Este tipo de planejamento computacional foi criado visando a eliminar as deficiências do planejamento $2 \mathrm{D}^{(4)}$. Kijewski et al. ${ }^{(5)}$ demonstraram que os modelos aplicados nessas simulações devem ser personalizados para que seja obtido um protocolo de tratamento que produza um tratamento efetivo, que gere no tumor uma dose absorvida tal que o controle do crescimento do tumor seja alcançado e minimize ao máximo as doses nos tecidos adjacentes sadios.

Este artigo mostra uma técnica de conversão de imagens de tomografia computadorizada (TC) ou de ressonância magnética (RM) em modelo de voxels tridimensional para fins de dosimetria. Voxels são como os pixels (elementos de imagem) num espaço tridimensional. Este modelo pode ser uma representação personalizada do paciente, que é empregada na simulação do fenômeno de transporte de partículas, por exemplo com o código MCNP (Monte Carlo N-Particle). O SISCODES (sistema computacional para dosimetria por nêutrons e fótons baseado em métodos estocásticos) é o sistema computacional que implementa esta técnica de geração do modelo de voxels e efetua cálculos dosimétricos. Desenvolvido para Linux em $\mathrm{C}++$, PHP, Python e SQL, o SISCODES teve seu registro depositado no Instituto Nacional de Propriedade Intelectual (INPI), mediado pela Comissão de Inovação e Transferência Tecnológica (CITT) da Universidade Federal de Minas Gerais (UFMG). Este sistema vem sendo utilizado no grupo de pesquisa Núcleo de Radiações Ionizantes/Conselho Nacional de Desenvolvimento Científico e Tecnológico (NRI/CNPq) ${ }^{(\mathbf{6})}$ para criar modelos de voxels antropomórficos e antropométricos que podem ser inseridos no código MCNP para modelagem de braquiterapias e teleterapias, aplicadas a tumores de pulmão, pelve, coluna, cabeça, pescoço, dentre outros ${ }^{(7,8)}$. O SISCODES disponibiliza um modo rápido de produzir modelos de voxels personalizados de qualquer paciente. Os módulos já desenvolvidos do SISCODES são mostrados junto com casos exemplos de planejamento radioterápico.

\section{MATERIAIS E MÉTODOS}

O sistema computacional desenvolvido, chamado de SISCODES, é uma ferramenta para planejamento computacional tridimensional de tratamentos radioterápicos ou procedimentos radiológicos, fornecendo interfaces amigáveis que permitem que profissionais da área de saúde operem o sistema. Partindo de imagens tomográficas do paciente, o plano de tratamento é modelado e simulado. O SISCODES então mostra as doses absorvidas, por meio de curvas de isodoses superpostas ao modelo.
O SISCODES acopla o modelo tridimensional ao código MCNP5, que simula o protocolo de exposição à radiação ionizante. O sistema possui arquitetura cliente/ servidor, operando em três ambientes distintos: estação de trabalho; Internet; servidor. O servidor pode ser um supercomputador ou um cluster de microcomputadores e é usado para os cálculos estocásticos e gerência e armazenamento do banco de dados de informações nucleares. A Internet permite que o servidor seja acessado por intermédio das estações de trabalho de, virtualmente, qualquer lugar do mundo. $\mathrm{E}$ a arquitetura cliente/servidor permite que o hardware necessário seja compartilhado entre vários grupos de pesquisa, dividindo custos e tornando o sistema ainda mais acessível.

O SISCODES é desenvolvido em módulos, ou subsistemas, com cada módulo operando independentemente. A arquitetura do sistema é mostrada na Figura 1. O SISCODES vem sendo usado em pesquisas de planejamento radioterápico desde 2003.

Para desenvolver o modelo do paciente utilizado na simulação são aplicados dois métodos distintos. Primeiro, um fantoma analítico com a biometria do paciente deve ser gerado por um código computacional acoplado ao SISCODES. Este código usa as medidas anatômicas do paciente para escalonar um fantoma padrão, de modo que este se adapte à biometria do paciente ${ }^{(9)}$.

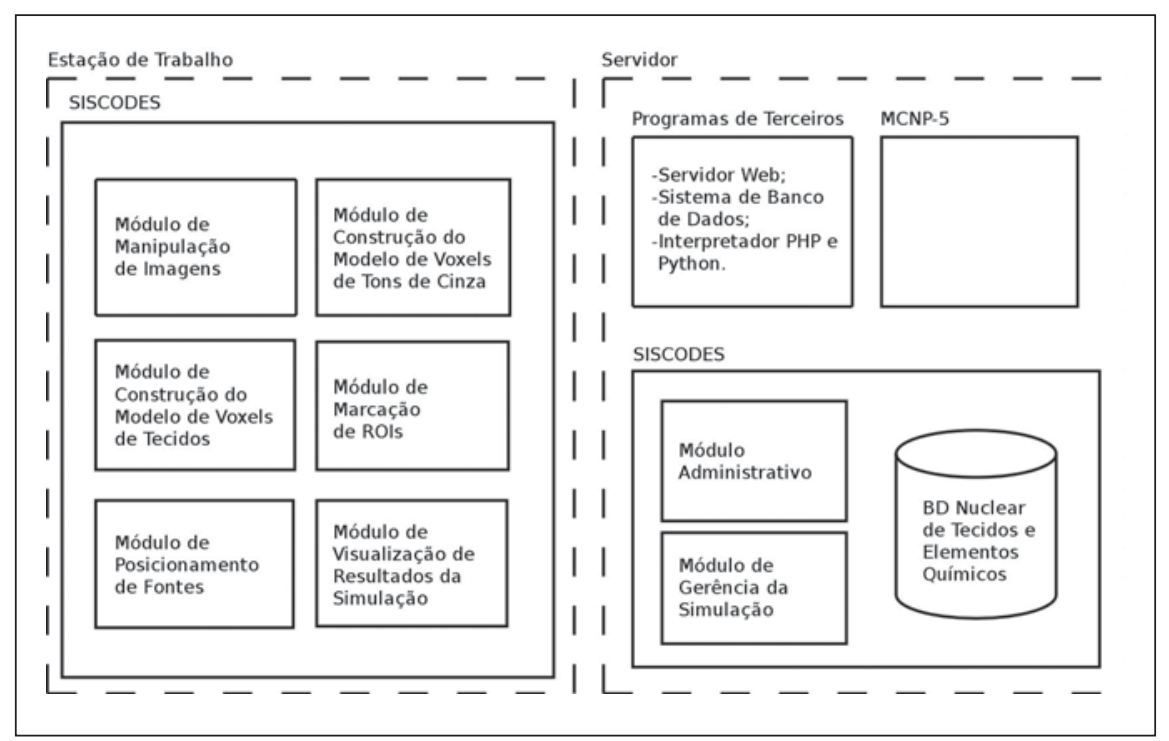

Figura 1. Arquitetura simplificada do SISCODES mostrando quais módulos executam no servidor e quais executam diretamente na estação de trabalho. 
Uma interface do SISCODES auxilia o usuário na seleção e entrada dos dados do paciente. O modelo analítico gerado é uma representação anatômica de corpo inteiro do paciente. Para a região de interesse, um modelo tridimensional de voxels é gerado. Este modelo é uma matriz de três dimensões, na qual cada elemento representa um tecido, equivalente ao tecido biológico correspondente. Este modelo representa fielmente a anatomia do paciente na região de interesse. A construção do modelo de voxels do paciente é feita em quatro etapas:

1. Digitalização de imagens tomográficas: imagens tomográficas em formato DICOM de TC ou RM são selecionadas, ou se necessário, digitalizadas de imagens radiológicas.

2. Tratamento das imagens: as imagens selecionadas são tratadas e recortadas para que se limitem à região de interesse. O módulo do SISCODES chamado de "Trata Imagem" é responsável por essa etapa.

3. Conversão da seleção de imagens do paciente numa matriz tridimensional baseada na escala de cinza das imagens: um modelo de voxels baseado nos tons de cinza das imagens do paciente é automaticamente gerado. O módulo do SISCODES chamado de "Gera Modelo de Cinza" é o responsável por gerar este modelo de voxels de tons de cinza, no qual é atribuído a cada elemento da matriz um valor entre 0 e 255 baseado no tom de cinza da imagem.

4. Conversão do modelo de tons de cinza no modelo de voxels baseado em tecidos: o módulo do SISCODES chamado de "Gera Modelo de Tecidos" permite ao usuário fazer a conversão do modelo obtido no passo 3 (modelo de voxel de tons de cinza) num modelo de voxels de tecidos, no qual cada voxel armazena a informação do tecido do paciente.

O SISCODES implementa e dá suporte a um banco de dados de informações nucleares de tecidos, que opera de modo independente da geração do modelo de voxels, embora seja utilizado por este. O módulo do SISCODES chamado de "Administrativo" permite o gerenciamento da informação de cada tecido, como a cor de representação, a composição química elementar, o kerma de fótons e nêutrons, o valor da eficiência biológica relativa (RBE), e outros fatores de ponderação, assim como de um link para a biblioteca nuclear de fótons e nêutrons Evaluated Nuclear Data File Bank - Edition 7 (ENDFB-7), utilizada no MCNP.

O módulo do SISCODES "Gera ROIs" permite a seleção dos voxels do modelo nos quais uma informação requerida (fluência, dose ou taxa de dose) é de interesse na simulação. Este módulo também permite a conversão dos arquivos no padrão do SISCODES para o formato do arquivo de entrada do MCNP. O modelo de voxels e o fantoma analítico devem ser unidos para que se efetue a completa simulação no MCNP. Após o término da simulação, o usuário executa o "Gera Saída", o módulo de saída do SISCODES. Este módulo converte a fluência de nêutrons ou fótons em dose usando os coeficientes de kerma versus fluência e mostra as curvas de isodoses, plotadas sobre o modelo de voxels do paciente. Um sumário do processo para a simulação de um planejamento radioterápico está ilustrado na Figura 2.

\section{Módulos básicos do SISCODES}

Módulo de manipulação de imagens - Neste módulo o usuário carrega as imagens tomográficas do paciente (TC ou RM) e, manipulando estas imagens, constrói um modelo de camadas, que nada mais é do que uma coleção de imagens agrupadas e cuja resolução foi padronizada. As imagens devem ser obtidas numa resolução tal que possa ser escalonada, reproduza fielmente a tomografia, e que este seja visível para o usuário. A resolução da imagem pode estar em torno de 100 ou 200 DPIs (pontos por polegada).

Módulo de geração do modelo de voxels de tons de cinza - Neste módulo o usuário carrega o modelo de camadas, define o tamanho de cada voxel, define o número de planos do modelo de voxels e, ajustando as imagens do modelo de camadas e a área correspondente ao modelo, constrói um modelo de voxels em tons de cinza.

As imagens do modelo de camadas podem ter sido digitalizadas em cores ou em escala de cinza. Se uma imagem colorida for usada, o tom de cinza armazenado num voxel será a média aritmética dos canais de cor (RGB - vermelho, verde, azul) dos pixels escolhidos para representar aquele voxel. Estudos foram executados para tentar identificar o algoritmo que escolha o pixel que melhor represente um determinado voxel. Entretanto, como o modelo de

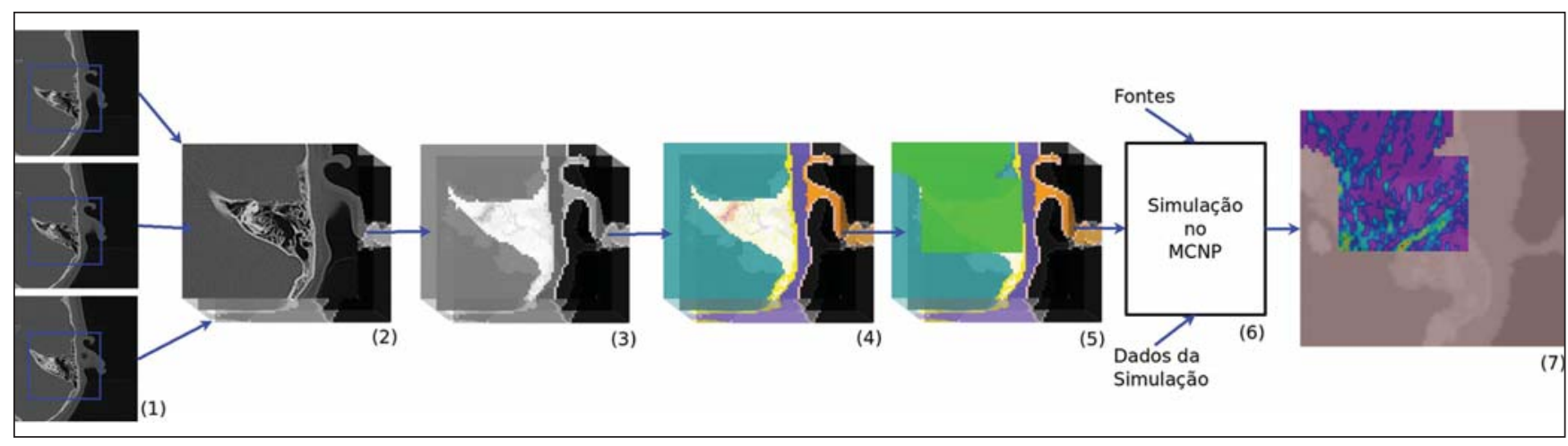

Figura 2. Processo simplificado da construção do modelo de voxels e simulação do tratamento radioterápico no SISCODES. Em resumo: imagens tomográficas são digitalizadas (1), convertidas em um modelo de camadas (2), convertidas em um modelo de voxels de tons de cinza (3) e em um modelo de voxels de tecidos (4). As ROls são marcadas no modelo (5). Este modelo é combinado com o fantoma paramétrico e com as informações das fontes radioativas. 0 arquivo resultante é simulado no MCNP (6). Após o término da simulação, o usuário pode visualizar as curvas de isodoses da distribuição espacial da dose absorvida (7). 
tons de cinza é usando apenas como referência para a construção do modelo de tecidos, optou-se por usar o tom de cinza do pixel central da área de imagem que representa o voxel, tornado o algoritmo de escolha computacionalmente eficiente.

Módulo de geração do modelo de voxels de tecidos - A interface que gera o modelo de voxels baseado em tecidos é mostrada na Figura 3A. O usuário carrega o modelo de tons de cinza gerado no módulo de geração do modelo de voxels de tons de cinza e aplica uma ferramenta de cor que altera individualmente cada voxel ou um conjunto deles, que pode ser selecionado pontualmente ou em grupos bi e tridimensionais. A seleção por grupos é feita pela semelhança de tons de cinza, cuja tolerância da variação pode ser ajustada. $\mathrm{O}$ usuário auxilia associando um tecido do banco de dados a cada voxel do modelo, identificando os órgãos e tecidos de interesse. O mesmo procedimento pode ser feito em modelos já construídos, de modo a alterar alguma parte do modelo.

Os tecidos são previamente registrados no banco de dados do SISCODES empregando-se o módulo administrativo. $\mathrm{O}$ voxel preenchido com um tecido recebe a cor associada a este tecido, permitindo ao usuário distinguir facilmente os voxels que já têm um tecido associado e os voxels ainda sem um tecido associado.

Módulo de seleção de ROIs - Este módulo funciona de maneira análoga ao módulo anterior, porém o usuário seleciona quais serão os voxels nos quais a fluência de nêutrons ou fótons será avaliada. Este passo é essencial, pois se a fluência for avaliada em todos os voxels do modelo o tempo de execução da simulação do planejamento radioterápico será inaceitável. Até o presente momento, com o uso deste módulo, o usuário pode gerar fragmentos do arquivo de entrada do MCNP, que representam o modelo de voxels, a composição química dos tecidos, as bibliotecas utilizadas e as solicitações de saída. Estes arquivos devem ser concatenados junto com o modelo analítico e as fontes e executados no MCNP. Após a execução, o SISCODES pode processar a saída gerada.

Módulo de gerenciamento - Este módulo, mostrado na Figura 3B, foi escrito em PHP (PHP Html Preprocessor) e deve ser

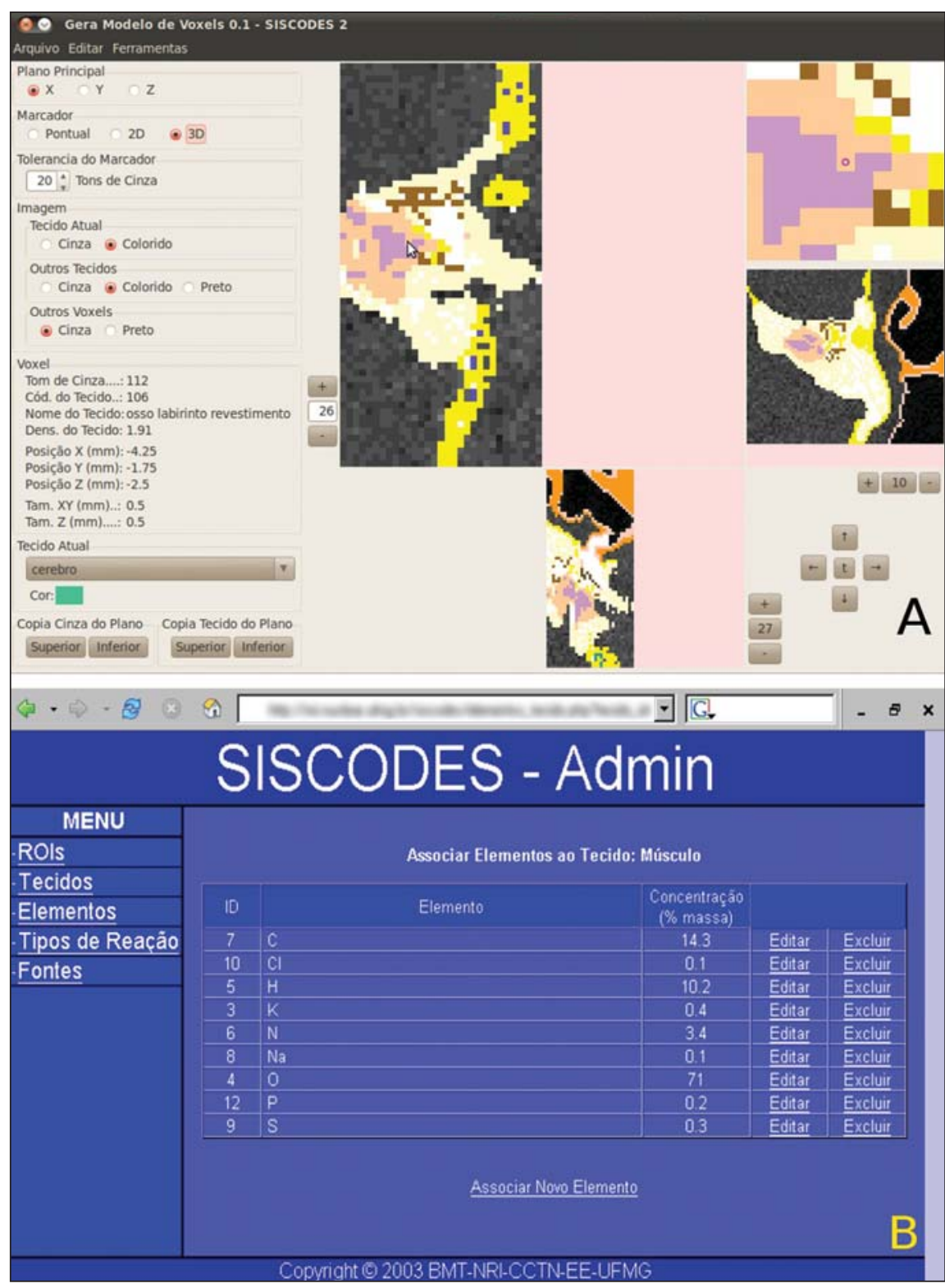

Figura 3. A: Interface do módulo de construção do modelo de voxels de tecidos mostrando a construção do modelo de voxels da região da orelha. B: Interface Web do módulo administrativo mostrando a página na qual é possível a edição da composição química dos tecidos e materiais.

acessado via um navegador de Internet. $\mathrm{O}$ usuário administrador pode incluir tecidos no banco de dados do SISCODES, junto com sua composição química e densidade de massa. O usuário administrador também pode incluir elementos químicos individuais no banco de dados do SISCODES. Cada elemento químico tem seus coeficientes de conversão de kerma para nêutrons e fótons cadastrados para um largo espectro de energia $(0,0025 \mathrm{eV}$ a $20 \mathrm{MeV})$. Podem também ser cadastrados coeficientes de RBE para reações nucleares específicas. Este módulo faz a interpolação dos coefi- cientes de kerma de cada elemento químico, dividindo o espectro em intervalos pequenos e regulares. Para que isso seja feito, a equação mostrada adiante é aplicada. Usando os coeficientes de kerma dos elementos químicos e a composição elementar em peso dos tecidos, este módulo calcula o kerma de cada tecido ou órgão cadastrado, considerando o coeficiente de kerma do elemento ponderado pela porcentagem de massa deste elemento no tecido. Para facilitar o cálculo da dose, apenas a fluência é avaliada na simulação no MCNP; desse modo pode ser calculado rapidamente o 
efeito de potencializadores de dose em função do tipo de partícula ou tipo de radiossensibilidade tecidual e da aplicação dos coeficientes de RBE, na dose aplicada.

$$
C=C a\left(\frac{E}{E a}\right)^{\left(\frac{\log (C p / C a)}{\log (E p / E a)}\right)}
$$

Esta equação é usada na interpolação dos coeficientes de kerma, onde: $\mathrm{C}$ - coeficiente de kerma a ser calculado; $\mathrm{Ca}-$ coeficiente de kerma anterior: o coeficiente conhecido de maior energia, imediatamente inferior ao que se quer calcular; $\mathrm{Cp}$ - coeficiente de kerma posterior: o coeficiente conhecido de menor energia, imediatamente posterior ao que se quer calcular; E - energia do coeficiente de kerma que se deseja calcular; Ea - energia do coeficiente de kerma anterior; Ep - energia do coeficiente de kerma posterior.

O SISCODES também permite o cadastro de fontes radioativas para braquiterapia ou teleterapia. $\mathrm{O}$ espectro radioativo de radionuclídeos ou de interações Bremsstrahlung podem ser incluídas no banco de dados do SISCODES, podendo ser associados a uma fonte externa ou interna.

Módulo de visualização de saída Este módulo, mostrado na Figura 4, lê o arquivo de saída gerado pelo MCNP, o modelo de voxels de tecidos e o arquivo com as ROIs, e mostra as curvas de isodoses sobrepostas ao modelo de voxels. A fluência em cada voxel, obtida da simulação no MCNP, e os coeficientes de kerma são usados no cálculo da dose absorvida (ver item 3). Para permitir uma boa visualização das isodoses sobre o modelo de voxels, o modelo é ampliado usando-se uma interpolação bicúbica e as isodoses são calculadas sobre este novo modelo por meio de uma interpolação isotrópica. O usuário pode controlar o nível de transparência da representação das isodoses, selecionando aquela que permite a melhor visualização.

\section{SISCODES - módulos avançados}

Módulo de posicionamento de fontes - Este módulo permite ao usuário, por intermédio de uma interface gráfica, selecionar e posicionar as fontes radioativas usadas na simulação. Nesta interface, tanto as fontes para braquiterapia quanto as para teleterapia são posicionadas. A posição é dada por um ponto arbitrário no modelo (definido por valores nos eixos X, Y e Z) e por uma orientação também arbitrária (definido por ângulos alfa - XY e beta - XZ). Para fontes de teleterapia também é necessário que sejam informadas a distância da fonte ao ponto de referência e as dimensões da janela.

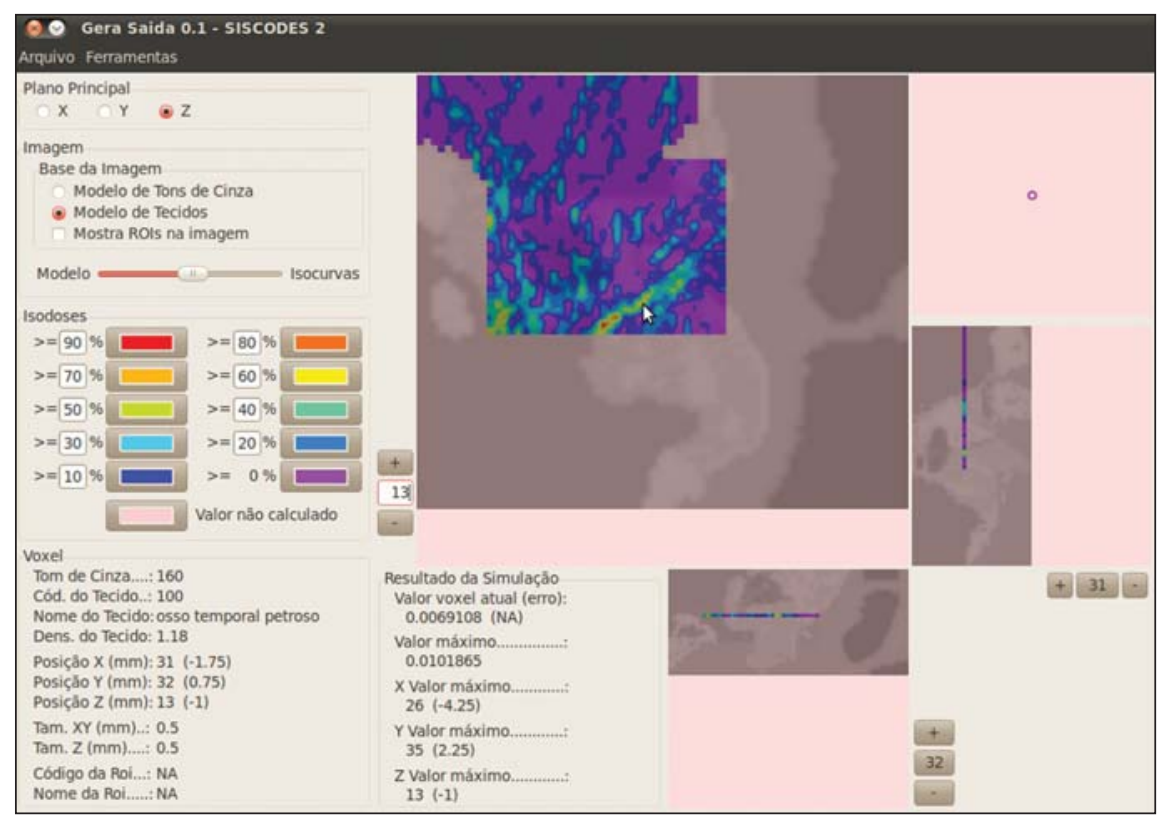

Figura 4. Interface do módulo de visualização dos dados de saída, mostrando as curvas obtidas como resultado de simulação sobre o modelo da região da orelha. Este módulo lê o modelo de voxels e a saída do MCNP e desenha as curvas de isodoses sobre a reconstrução de imagens do paciente.
Módulo de gerência da simulação Este módulo permite ao usuário montar o arquivo completo de entrada do MCNP e gerenciar a execução da simulação. O usuário seleciona os arquivos necessários para a simulação, criando um grupo. O módulo então monta o arquivo de entrada do MCNP, envia o arquivo para a simulação e recebe o resultado após execução.

\section{Cálculo de dose no SISCODES}

O SISCODES trabalha como uma interface para o código MCNP. O MCNP é um código nuclear baseado em processos estocásticos, no qual o trajeto de cada partícula radioativa gerada é rastreado, prevendo as interações desta com a matéria. O histórico de cada partícula é então memorizado. No SISCODES o modelo de voxels é convertido num arquivo de entrada do MCNP, que então simula a interação das partículas nucleares com os elementos químicos presentes nos tecidos associados a cada voxel.

O MCNP retorna para o SISCODES o número de partículas incidentes por unidade de área de cada voxel (fluência) normalizado pelo número de partículas emitidas pela fonte. A fluência gerada na simulação no MCNP é transformada em dose absorvida multiplicando-se a mesma pelos coeficientes de conversão fluência-kerma adequados, pela surgência da fonte (número de partículas emitidas por unidade de tempo) e pelo tempo de exposição. Assim, o SISCODES pode calcular a dose absorvida em cada voxel e, se necessário, aplicar a esta dose os coeficientes de RBE, obtendo uma dose biologicamente ponderada. O RBE é definido para cada tecido para cada reação nuclear e é cadastrado no banco de dados do SISCODES através do módulo administrativo.

O MCNP pode calcular diretamente a dose absorvida ou a taxa de dose e transferir estes valores para o SISCODES. Entretanto, o SISCODES trabalha com a fluência e converte esta para dose absorvida ou dose ponderada biologicamente, de acordo com o interesse do usuário. Isto se deve ao fato de que radioprotetores ou radiopotencializadores podem afetar a dose absorvida, entretanto, não afetam a fluência. A terapia por captura de nêutrons pelo boro (BNCT) pode ser citada como exemplo de avaliação 
que envolve a necessidade de potencializadores. Aplicando um sistema de conversão fluência $\rightarrow$ dose, o sistema pode calcular o impacto do uso destes protetores/potencializadores rapidamente, já que o MCNP é executado apenas uma vez.

\section{RESULTADOS}

O SISCODES vem sendo utilizado no grupo de pesquisa NRI do Departamento de Engenharia Nuclear da UFMG (DEN/ UFMG) para a construção de vários modelos de voxels representando regiões anatômicas de seres humanos e pequenos animais. Alguns destes modelos foram aplicados na simulação de tratamentos por braquiterapia ou teleterapia. Nesta sessão serão mostrados alguns destes modelos e resultados de algumas simulações.

\section{Tórax humano feminino}

Este modelo foi construído no NRI e é mostrado nas Figuras $5 \mathrm{~A}$ e $5 \mathrm{~B}^{(\mathbf{1 0 )}}$. Ele foi desenvolvido lado a lado com um fantoma antropométrico e antropomórfico real, para investigar tratamento para câncer de mama, esôfago e pulmão. Este fantoma pode ser bastante útil na verificação da precisão do SISCODES, mediante comparação das doses absorvidas avaliadas em experimentos no fantoma real com as obtidas pela simulação computacional.

O câncer de mama é particularmente difícil de irradiar em razão da sua proximidade com os pulmões, que são muito radiossensíveis. Este modelo pode auxiliar no desenvolvimento de melhores protocolos em radioterapias para câncer de mama, permitindo selecionar aqueles que produzem menores doses nos pulmões e maiores doses no tumor.

\section{Pelve humana masculina}

Este modelo foi desenvolvido no SISCODES para permitir estudos em câncer de próstata $^{(7)}$. Assim, com o modelo de tórax feminino este modelo tem seu par real, permitindo seu uso na verificação da acuidade do SISCODES. O modelo de voxels foi desenvolvido a partir de imagens do projeto Visible Human ${ }^{(11)}$, que também serviram de base para o fantoma real, construído com material tecido-equivalente. $\mathrm{O}$ câncer de próstata é um dos tipos de cân- cer mais comuns em homens. Este modelo pode ser usado na simulação de protocolos de teleterapia e radioterapia. Uma secção deste modelo é apresentada na Figura 5C.

\section{Orelha humana}

Este modelo representa a região da orelha humana, como mostrado na Figura 6. Foi desenvolvido num projeto de pesquisa de mestrado, com o objetivo de estudar os efeitos colaterais após teleterapia de cabeça e pescoço ${ }^{(\mathbf{1 2})}$. O conhecimento da dose absorvida real é relevante por dois fatores: a) para se saber qual nível de dose causa efeitos colaterais na orelha; b) para auxiliar na escolha do melhor protocolo para cada janela de irradiação, evitando a exposição da orelha. Dois protocolos de tratamento foram simulados e apresentaram grande heterogeneidade de dose no osso
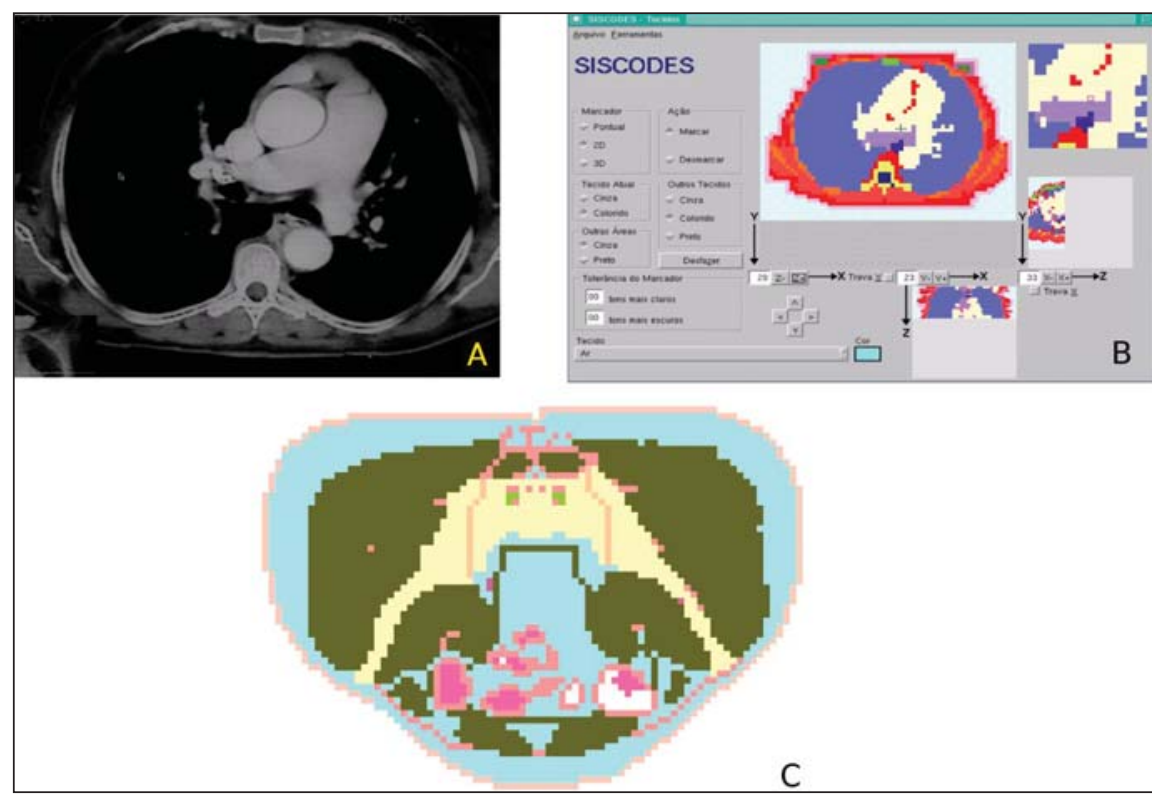

Figura 5. Criação do modelo de voxels do tórax. A: Imagem digitalizada de TC do tórax. B: Interface antiga de criação do modelo de voxels de tecidos do SISCODES mostrando o modelo no mesmo plano da imagem. C: Modelo de voxels de pelve masculina.

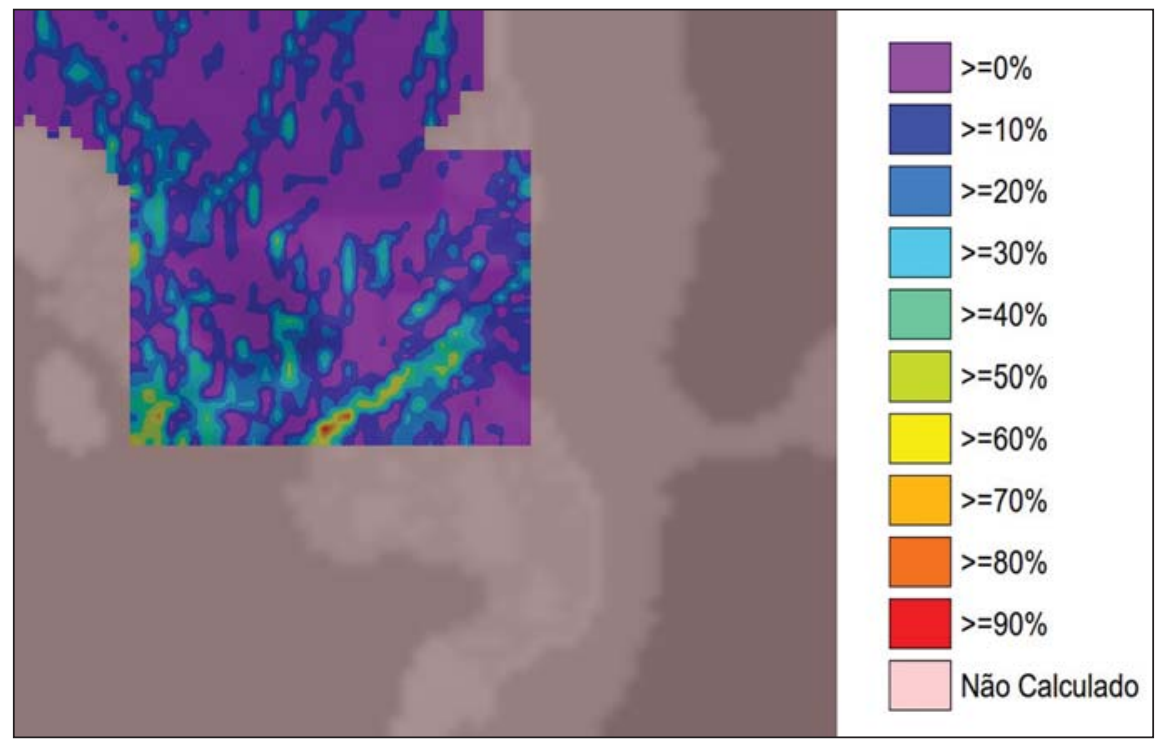

Figura 6. Curvas de isodoses na região da orelha, com a respectiva escala. As doses mostradas são absorvidas devido a um protocolo de irradiação da orelha. Notar a grande heterogeneidade da dose, que se contrapõe às doses suavemente distribuídas de um fantoma de água. 
petroso, que pode ser associada à heterogeneidade do tecido e ao espalhamento de fótons. A heterogeneidade da dose observada não pode ser modelada nos fantomas de água usadas em centros radioterápicos para calibração das curvas de isodoses. A aplicação de métodos homogêneos para o cálculo da dose pode produzir super ou subestimação da dose nas estrutura da orelha.

\section{Cabeça de criança}

Este modelo foi construído para estudo de implantes braquiterápicos de sementes de I-125 ou sementes cerâmicas tipo bioglass dopado com Sm-153 ou Ho-166. Este tipo de implante pode ser uma opção aos implantes de I-125 ${ }^{(\mathbf{1 3}, \mathbf{1 4})}$. As curvas de isodose mostradas na Figura 7A representam as doses produzidas pelos raios gama emitidos por sementes de Sm-153 implantadas.

\section{Fígado de coelho}

No NRI ${ }^{(\mathbf{1 5 , 1 6 )}}$ foram desenvolvidos modelos de partes de pequenos animais, como cérebro e abdome de coelho. Estes modelos proporcionam a dosimetria experimental e computacional em estudos com pequenos animais. Neste caso, o modelo computacional auxilia a possibilidade de redução do número de cobaias em experimentação animal. A avaliação dosimétrica neste caso teve como objetivo estimar a distribuição espacial da energia das partículas beta e gama, produzidas por fontes de braquiterapia produzidas por sementes cerâmicas radioativas. Para fazer este estudo, um modelo de voxels da região do tórax e abdome de coelho foi desenvolvido e simulações dos implantes radioativos foram executadas. As curvas de isodoses obtidas são mostradas na Figura 7B.

\section{Cérebro de coelho}

Um modelo de voxels da cabeça de um coelho foi construído por Costa para efetuar um estudo de implantes de bioglass no cérebro $^{(17)}$. Os implantes consistem em 15 sementes de bioglass Ca-Si com Sm-153 incorporado $^{(\mathbf{1 8 - 2 1 )}}$. Um modelo de voxels foi construído no SISCODES, baseado em imagens de TC de cobaia. A simulação neste estudo mostra a distribuição espacial da taxa de dose no cérebro do coelho decorrente dos implantes de bioglass Sm153. As curvas de isodoses obtidas na simulação são mostradas na Figura 7C.

\section{DISCUSSÃO}

O sistema computacional apresentado vem sendo usado pelo grupo de pesquisa NRI na criação de fantomas computacionais antropomórficos e antropométricos baseados em modelos de voxels e na simulação de tratamentos radioterápicos. $\mathrm{O}$ sistema está sendo aplicado no estudo do transporte de partículas nucleares no tratamento de câncer de mama, pulmão, colo uterino, próstata, coluna vertebral, cabeça e pescoço, dentre outros.

A interface gráfica provida pelo SISCODES permite que o MCNP seja usado por profissionais que não teriam acesso a este código devido à complexidade da sintaxe de seu arquivo de entrada, assim como capacidade do módulo de visualização de saída de extrair os resultados da simulação e representar estes resultados em imagens.

Os modelos desenvolvidos e os dados armazenados no banco de dados, juntamente com o SISCODES, formam uma base para execução de simulações de radioterapias. Esta base permite o desenvolvimento de estudos de tratamentos radioterápicos com eficiência, em termos de velocidade de pesquisa, muito superior à conseguida sem ela, e com a precisão da dosimetria via MCNP.

Os resultados das simulações de tratamentos mostram que a dose absorvida apresenta grande heterogeneidade espacial nas regiões do corpo onde ocorrem grandes variações de massa ou densidade, tal qual ossos, cavidades aéreas e tecidos de baixa densidade como os pulmões inflados, possivelmente divergindo dos valores obtidos em simuladores homogêneos de água e mesmo de valores de dose encontrados por métodos analíticos complexos. Esta representação da heterogeneidade na dose apenas pode ser obtida por simulação via MCNP, pois métodos analíticos não conseguem lidar corretamente com as variações encontradas nos tecidos humanos, principalmente em regiões de interface ${ }^{(\mathbf{2 2 , 2 3})}$.

\section{CONCLUSÕES}

O sistema permite construir um modelo de voxels personalizado do paciente em um tempo relativamente curto, tornando o planejamento radioterápico mais preciso quando se empregam códigos estocásticos tipo MCNP. A representação biométrica do

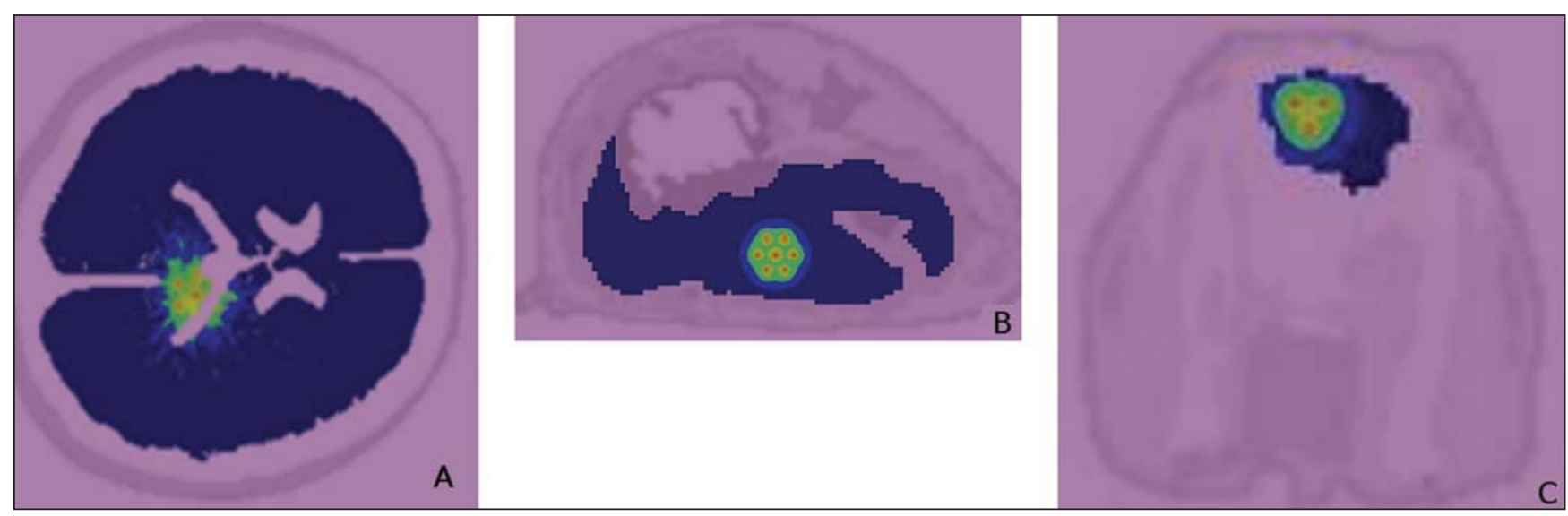

Figura 7. A: Curvas de isodoses obtidas na simulação de implantes de sementes de bioglass com Sm-153 em cérebro de criança. B: Curvas de isodoses geradas por implantes de sementes cerâmicas em fígado de coelho. C: Curvas de isodoses geradas por implantes de sementes de bioglass em cérebro de coelho. 
paciente, junto com a região de irradiação fielmente representada pelo modelo de voxels, torna a simulação mais específica e personalizada. A combinação da simulação via MCNP com um modelo personalizado do paciente traz grandes melhorias na dosimetria de tratamentos radioterápicos, o que deve contribuir para o aumento da efetividade do tratamento, com diminuição da morbidade deste. Os autores desejam que estes estudos possam colaborar, ampliar e melhorar os procedimentos de avaliação dosimétrica presentes na radioterapia e radiologia.

\section{Agradecimentos}

Os autores agradecem à Coordenação de Aperfeiçoamento de Pessoal de Nível Superior (Capes) e ao Conselho Nacional de Desenvolvimento Científico e Tecnológico $(\mathrm{CNPq})$, pelo suporte e apoio à pesquisa no grupo Núcleo de Radiações Ionizantes da Universidade Federal de Minas Gerais (NRI-UFMG).

\section{REFERÊNCIAS}

1. Emami B. Oral cavity. In: Perez CA, Brady L, editors. Principles and practice of radiation oncology. 3rd ed. Philadelphia, PA: LippincottRaven; 1998. p. 981-1002.

2. MCNP-X-5 Monte Carlo Team. A general Monte Carlo N-particle transport code manual, version 5. Los Alamos, NM: Los Alamos National Laboratory, 2003.

3. Purdy JA. Three-dimensional physics and treatment planning. In: Perez CA, Brady LW, editors. Principles and practice of radiation oncology. $3 \mathrm{rd}$ ed. Philadelphia, PA: Lippincott-Raven; 1998. p. 343-70

4. Emami B, Graham MV, Michalski JM, et al. Three-dimensional conformal radiation therapy: clinical aspects. In: Perez CA, Brady LW, editors. Principles and practice of radiation oncology. 3rd ed. Philadelphia, PA: Lippincott-Raven; 1998. p. 371-86.

5. Kijewski P. Three-dimensional treatment planning. In: Mauch PM, Loeffler JS, editors. Radiation oncology: technology and biology. Philadelphia, PA: Saunders; 1994. p. 10-33.

6. NRI - Núcleo de Radiações Ionizantes. Página inicial. [acessado em $1^{\circ}$ de fevereiro de 2011]. Disponível em: http://nri.nuclear.ufmg.br

7. Silva CHT, Campos TPR. Fantoma computacional de voxel, associado a fantoma real antropomórfico antropométrico, para dosimetria. In: International Nuclear Atlantic Conference; 2000; Rio de Janeiro, RJ, Brasil. ABEN - Associação Brasileira de Energia Nuclear. Anais. 2000. p. 17.

8. Silveira MF, Campos TPR. Implantação de compostos radioativos para controle de tumores ósseos nos membros. In: $4^{\circ}$ Congresso Latino-Americano de Órgãos Artificiais e Biomateriais; 2006; Caxambu, MG, Brasil. Anais. 2006. p. 1-7.

9. Mata PHC, Trindade BM, Campos TPR. Fantoma parametrizado com órgãos em voxels inseridos para oncologia. In: $3^{\circ}$ Congresso Latino Americano de Órgãos Artificiais e Biomateriais; 2004; Campinas, SP, Brasil. Anais. 2006. p. 1-6.

10. Maia M. Fantoma antropomórfico antropométrico de tórax para fins de radioproteção e dosimetria [dissertação]. Belo Horizonte, MG: Universidade Federal de Minas Gerais; 2004.

11. VHP. The Visible Human Project Overview. [acessado em 14 de setembro de 2010]. Disponível em: http://www.nlm.nih.gov/research/visible/ visible_human.html

12. Trindade DFM. Efeitos deletérios induzidos por exposição indireta do aparelho auditivo durante radioterapia de cabeça e pescoço - correlacionamento dosimétrico [dissertação]. Belo Horizonte, MG: Universidade Federal de Minas Gerais; 2006.

13. Duarte IL, Campos TPR. Avaliação dosimétrica clínica de Implantes de biovidros incorporando Sm-153 para tratamento de tumores cerebrais. In: $4^{\circ}$ Congresso Latino Americano de Órgãos Artificiais e Biomateriais; 2006; Caxambu, MG, Brasil. Anais. 2006. p. 1-6.

14. Thompson L, Campos TPR. Fantoma real de cabeça e pescoço desenvolvido para avaliações radiodosimétricas. In: $4^{\circ}$ Congresso Latino Americano de Órgãos Artificiais e Biomateriais; 2006; Caxambu, MG, Brasil. Anais. 2006. p. 1-8.

15. Andrade JPL, Campos TPR. Avaliação da energia específica absorvida gerada de biovidros radioativos implantados em fígado em modelo animal. In: $4^{\circ}$ Congresso Latino Americano de Órgãos Artificiais e Biomateriais; 2006; Caxambu, MG, Brasil. Anais. 2006. p. 1-10.

16. Campos TPR, Andrade JPL, Costa IT, et al. Estudo preliminar da degradação de sementes de Sm-153 aplicadas e implantes de fígado de coelho. In: $4^{\circ}$ Congresso Latino Americano de Órgãos Artificiais e Biomateriais; 2006; Caxambu, MG, Brasil. Anais. 2006. p. 1-6.

17. Costa IT, Campos TPR. Resposta radiodosimétrica de implantes de sementes de biovidros radioativos no cérebro de coelhos. In: $4^{\circ}$ Congresso Latino Americano de Órgãos Artificiais e Biomateriais; 2006; Caxambu, MG, Brasil. Anais. 2006. p. $1-7$.

18. Mendes BM, Campos TPR. Investigation of the specific energy deposition form radionuclidehydroxyapatite macroaggregate in brain interstitial implants. Key Engineering Materials. 2004; 254:83-6.

19. Mendes BM, Campos TPR. Ecogenic response and dosimetric evaluation of hydroxyapatite macroaggregates implants. In: COBEM $2005-18$ th International Congress of Mechanical Engineering; 2005; Rio de Janeiro, RJ, Brasil. Anais. 2005. p. $1-8$.

20. Roberto WS, Pereira MM, Campos TPR. Analysis of bioactive glasses obtained by sol-gel processing for radioactive implants. Materials Research. 2003;6:123-7.

21. Roberto WS, Pereira MM, Campos TPR. Struc ture and dosimetric analysis of biodegradable glasses for prostate cancer treatment. Artif Organs. 2003;27:432-6.

22. Van Esch A, Tillikainen L, Pyykkonen J, et al Testing of the analytical anisotropic algorithm for photon dose calculation. Med Phys. 2006;33: 4130-48.

23. Carrasco P, Jornet N, Duch MA, et al. Comparison of dose calculation algorithms in slab phantoms with cortical bone equivalent heterogeneities. Med Phys. 2007;34:3323-33. 